Pacific

Journal of

Mathematics

\title{
LOCAL REFLEXIVITY OF DUAL BANACH SPACES
}

Manuel González and Antonio Martínez-Abejón 


\title{
LOCAL REFLEXIVITY OF DUAL BANACH SPACES
}

\author{
Manuel GonzÁlez and Antonio Martínez-ABejón
}

We introduce a notion of finite representability of dual Banach spaces in their subspaces preserving duality (f.d.-r in short) which arises in a natural way in situations such as the principle of local reflexivity. We give a characterization for the f.d.-r. which yields a version of the principle of local reflexivity, and can be applied to the study of the duality theory for ultrapowers of operators. For example, we show that the kernel $\operatorname{ker}\left(T^{* *} \mathfrak{U}\right)$ of an ultrapower of the second conjugate of an operator $T$ is finitely representable in $\operatorname{ker}\left(T_{\mathfrak{U}}\right)$, and $\operatorname{ker}\left(T_{\mathfrak{U}}{ }^{*}\right)$ is f.d.-r. in $\operatorname{ker}\left(T^{*} \mathfrak{U}\right)$. Moreover, we study the duality properties of three semigroups of operators related with the superreflexivity and the finite representability of $c_{0}$ and $\ell_{1}$ in a Banach space.

\section{Introduction.}

We introduce the concept of finite representability preserving duality (f.d.-r. in short) of a Banach space in its subspaces, and the polar property and the $\mathcal{A}$-polar property for subspaces of a dual Banach space, where $\mathcal{A}$ stands for a class of operators. Given a subspace $Z$ of a dual space $X^{*}$, we show in Theorem 4 that $X^{*}$ is f.d.-.r. in $Z$ if and only if $Z$ has the polar property. As a consequence, if a subspace $Z$ of a dual space $X^{*}$ has the polar property, then $Z$ is norming on $X$, but the converse implication is not true.

We give some consequences of the $\mathcal{A}$-polar property in Theorem 7 , from which it follows a version of the principle of local reflexivity: $X^{* *}$ is f.d.-r. in $X[8]$, in such a way that given an operator $T \in \mathcal{B}(X, Y)$ we obtain estimates for the norm of the restrictions of $T^{* *}$ to finite dimensional subspaces in terms of the norm of the corresponding restrictions of $T$.

In Section 3 we apply these results to the study of the duality theory for ultrapowers of operators. From Theorem 4 it easily follows that the dual space $X_{\mathfrak{U}}{ }^{*}$ of the ultrapower $X_{\mathfrak{U}}$ of $X$ is f.d.-r. in $X^{*} \mathfrak{U}$, a result first proved by Heinrich [5]. Moreover, the general character of our result allows us to obtain some consequences which do not follow from the principle of local reflexivity or the results of [5]. Namely, in Theorem 11 we show that for every $T \in \mathcal{B}(X, Y)$ the kernel of the conjugate operator $T_{\mathfrak{U}}{ }^{*}$ is f.d.-r. in the 
kernel of $T^{*} \mathfrak{U}$, where $T_{\mathfrak{U}}$ is an ultrapower of $T$, and the $\operatorname{kernel} \operatorname{ker}\left(T^{* *} \mathfrak{U}\right)$ is finitely representable in $\operatorname{ker}\left(T_{\mathfrak{U}}\right)$.

We study the semigroups $\mathcal{W}^{u p}{ }_{+}, \mathcal{U}^{u p}{ }_{+}$and $\mathcal{R}^{u p}{ }_{+}$, introduced in $[\mathbf{1 3}, 4]$. Denoting any of them by $\mathcal{A}_{+}$, we show that an operator $T$ belongs to $\mathcal{A}_{+}$if and only if the second conjugate $T^{* *}$ belongs to $\mathcal{A}_{+}$. Moreover, $T_{\mathfrak{U}}{ }^{*} \in \mathcal{A}_{+}$ if and only if $T^{*} \mathfrak{U} \in \mathcal{A}_{+}$. Observe that $T_{\mathfrak{U}}{ }^{*}$ is an extension of $T^{*} \mathfrak{U}$. Finally, we give a proof of the fact, first proved in [13], that $T^{*} \in \mathcal{W}^{u p}+$ if and only if $\operatorname{ker}\left(T_{\mathfrak{U}}{ }^{*}\right)=\operatorname{ker}\left(T^{*} \mathfrak{U}\right)$.

We use standard notations: $X$ and $Y$ are Banach spaces, $B_{X}$ the closed unit ball of $X$, and $S_{X}$ the unit sphere of $X$. The class of (bounded linear) operators from $X$ to $Y$ is $\mathcal{B}(X, Y)$, the dual of $X$ is $X^{*}$, and given an operator $T \in \mathcal{B}(X, Y)$, we denote by $T^{*}: Y^{*} \longrightarrow X^{*}$ its conjugate operator, and by $R(T), \operatorname{ker}(T)$ and $\operatorname{coker}(T):=Y / \overline{R(T)}$ the range, the kernel and the cokernel of $T$. Observe that coker $(T)^{*}$ can be identified with $\operatorname{ker}\left(T^{*}\right)$.

Given a class of operators $\mathcal{A}$ and a pair of Banach spaces $X$ and $Y$, we denote by $\mathcal{A}(X, Y)$ the component $\mathcal{A} \cap \mathcal{B}(X, Y)$ of all operators of $\mathcal{A}$ between $X$ and $Y$. Also, $F^{\perp}$ is the annihilator of a subspace $F$, and $\mathbb{N}$ is the set of all positive integers. We identify $X$ with a subspace of $X^{* *}$. Given a subset $A$ of $X$, we denote by $\langle A\rangle$ the closure of the span of $A$ in $X$.

The letters $\mathfrak{U}, \mathfrak{V}, \ldots$ will be reserved to denote ultrafilters. An ultrafilter $\mathfrak{U}$ on a set $I$ is said to be countably incomplete if there is a countable partition $\left\{I_{n}: n \in \mathbb{N}\right\}$ of $I$ such that for every positive integer $n$, we have that $I_{n} \notin \mathfrak{U}$. Every infinite set admits a countably incomplete ultrafilter. Throughout the paper, we assume that the ultrafilters are countably incomplete.

Given a number $0<\varepsilon<1$, an operator $T \in \mathcal{B}(X, Y)$ is said to be an $\varepsilon$-isometry if $(1+\varepsilon)^{-1}<\|T x\|<1+\varepsilon$ for all $x \in S_{X}$, and if the $\varepsilon$-isometry $T$ is onto, then $X$ is said to be $\varepsilon$-isometric to $Y$. A Banach space $X$ is said to be finitely representable in $Y$ if given $\varepsilon>0$ and a finite dimensional subspace $M$ of $X$ there exists an $\varepsilon$-isometry $T: M \longrightarrow Y$. We will write $X$ f.r. in $Y$ to mean that the space $X$ is finitely representable in $Y$. Given a number $C>1$, two sequences $\left(x_{n}\right)$ and $\left(y_{n}\right)$ are said to be $C$-equivalent if for every sequence $\left(a_{k}\right)$ of scalars and every $n$ we have

$$
C^{-1}\left\|\sum_{k=1}^{n} a_{k} x_{k}\right\| \leq\left\|\sum_{k=1}^{n} a_{k} y_{k}\right\| \leq C\left\|\sum_{k=1}^{n} a_{k} x_{k}\right\| .
$$

\section{Finite representability preserving the duality.}

Since we need to use ultrapowers, we recall here some definitions and results, and refer to [5] for more information. Let $I$ be an infinite set. We denote by $\ell_{\infty}(I, X)$ the Banach space of bounded families $\left(x_{i}\right)_{i \in I}$ in $X$ with norm $\left\|\left(x_{i}\right)\right\|:=\sup \left\{\left\|x_{i}\right\|: i \in I\right\}$. 
Let $\mathfrak{U}$ be an ultrafilter on $I$ and let $N_{\mathfrak{U}}(X)$ be the closed subspace of all families $\left(x_{i}\right) \in \ell_{\infty}(I, X)$ which converge to 0 following $\mathfrak{U}$. The ultrapower of $X$ following $\mathfrak{U}$ is defined as follows:

$$
X_{\mathfrak{U}}:=\frac{\ell_{\infty}(I, X)}{N_{\mathfrak{U}}(X)} .
$$

The element of $X_{\mathfrak{U}}$ admitting the family $\left(x_{i}\right) \in \ell_{\infty}(I, X)$ as a representative is denoted by $\left[x_{i}\right]_{i}$, or simply $\left[x_{i}\right]$ if it does not lead to confusion. The norm of $\left[x_{i}\right]$ in $X_{\mathfrak{U}}$ is given by

$$
\left\|\left[x_{i}\right]\right\|=\lim _{\mathfrak{U}}\left\|x_{i}\right\| .
$$

The ultrapower $X_{\mathfrak{U}}$ canonically contains an isometric copy of $X$ generated by the constant families of $\ell_{\infty}(I, X)$. We identify this copy with $X$. An operator $T \in \mathcal{B}(X, Y)$ admits an extension $T_{\mathfrak{U}} \in \mathcal{B}\left(X_{\mathfrak{U}}, Y_{\mathfrak{U}}\right)$ given by $T_{\mathfrak{U}}\left[x_{i}\right]:=\left[T x_{i}\right]$.

The ultrapower $\left(X^{*}\right)_{\mathfrak{U}}$ is canonically contained in $\left(X_{\mathfrak{U}}\right)^{*}$, but in general these spaces do not coincide. Actually, given $\left(f_{i}\right) \in \ell_{\infty}\left(I, X^{*}\right)$, the class $\left[f_{i}\right] \in X^{*} \mathfrak{U}$ is identified with the element $f \in\left(X_{\mathfrak{U}}\right)^{*}$ given by $f\left(\left[x_{i}\right]\right):=$ $\lim _{\mathfrak{U}} f_{i}\left(x_{i}\right)$. Heinrich [5] proved that $\left(X^{*}\right)_{\mathfrak{U}}$ coincides with $\left(X_{\mathfrak{U}}\right)^{*}$ if and only if $X$ is superreflexive.

In the following definition we introduce a concept of finite representability which is stronger than the usual one: The finite representability preserving the duality.

Definition 1. Let $Z$ be a subspace of the dual $X^{*}$ of a Banach space $X$. We say that $X^{*}$ is finitely representable in $Z$ preserving the duality (f.d.-r. in short) if for every couple of finite dimensional subspaces $F$ of $X^{*}$ and $G$ of $X$, and for every $0<\varepsilon<1$, there is an $\varepsilon$-isometry $L: F \longrightarrow Z$ such that $(L f)(x)=f(x)$ for all $x \in G$ and all $f \in F$.

The celebrated principle of local reflexivity states that the second dual $X^{* *}$ is f.d.-r. in $X$. We refer to [10] for an elementary proof of this principle.

A subspace $Z$ of a dual space $X^{*}$ is said to be norming if for every $x \in$ $X$ we have that $\|x\|=\sup \left\{f(x): f \in B_{Z}\right\}$. It easily follows from the Hahn-Banach theorem that a subspace $Z$ of $X^{*}$ is norming if and only if ${\overline{B_{Z}}}^{\sigma\left(X^{*}, X\right)}=B_{X^{*}}($ see $[\mathbf{1}])$.

Let $X$ be a Banach space and $k, l$ two positive integers. A linear function $f: \mathbb{R}^{k} \longrightarrow \mathbb{R}^{l}$, represented by a matrix $\left(a_{i j}\right)_{i=1 j=1}^{l}$, induces an operator

$$
f_{X}: X \times \stackrel{k}{\cdots} \times X \longrightarrow X \times \cdots+\cdots \times X
$$

in a natural way $f_{X}\left(x_{i}\right):=\left(\sum_{j=1}^{k} a_{i j} x_{j}\right)$. Note that $\left(f_{X}\right)^{*}=f^{*} X^{*}$. We denote by $\mathcal{L}$ the class of all these operators.

$$
\mathcal{L}:=\left\{f_{X}: k, l \in \mathbb{N}, f: \mathbb{R}^{k} \longrightarrow \mathbb{R}^{l} \text { linear, } X \text { Banach space }\right\} .
$$


Henceforth we will denote by $\ell_{1}^{k}(X)$ and $\ell_{\infty}^{k}(X)$ the space $X \times \cdots \times X$ endowed with the norms $\left\|\left(x_{j}\right)_{j=1}^{k}\right\|:=\sum_{j=1}^{k}\left\|x_{j}\right\|$ and $\left\|\left(x_{j}\right)_{j=1}^{k}\right\|:=$ $\sup _{1 \leq j \leq k}\left\|x_{j}\right\|$, respectively. Given two subsets $A \subset X$ and $B \subset X^{*}$, their polar sets are defined as follows:

$$
\begin{gathered}
A^{\circ}:=\left\{f \in X^{*}:|f(a)| \leq 1 \text { for all } a \in A\right\} ; \\
B_{\circ}:=\{z \in X:|f(z)| \leq 1 \text { for all } f \in B\} .
\end{gathered}
$$

Note that the sets $A^{\circ}$ and $B_{\circ}$ are closed in the norm topology. If, in addition, $A$ and $B$ are convex and symmetric then we have $\left(B_{\circ}\right)^{\circ}=\bar{B}^{\sigma\left(X^{*}, X\right)}$ and $\left(A^{\circ}\right)_{\circ}=\bar{A}$.

Definition 2. A subspace $Z$ of a dual space $X^{*}$ is said to have the polar property if for every $k, l$ in $\mathbb{N}$ and every linear function $f: \mathbb{R}^{k} \longrightarrow \mathbb{R}^{l}$ we have

$$
\overline{f_{X}\left(B_{\ell_{1}^{k}(X)}\right)}=\left(\left.f^{*}{ }^{*}\right|_{\ell_{\infty}^{l}(Z)} ^{-1} B_{\ell_{\infty}^{k}(Z)}\right)_{\circ} .
$$

Observe that the inclusion $\overline{f_{X}\left(B_{\ell_{1}^{k}(X)}\right)} \subset\left(\left.f^{*}{ }_{X^{*}}\right|_{\ell_{\infty}^{l}(Z)} ^{-1} B_{\ell_{\infty}^{k}(Z)}\right)_{\circ}$ is always true. So, taking into account that the identities $\overline{T\left(B_{X}\right)}=\left(T^{*-1} B_{X^{*}}\right)_{\circ}$, $T^{*-1} B_{X^{*}}=\left(T B_{X}\right)^{\circ}$ hold for every operator $T \in \mathcal{B}(X, Y)$, we obtain the following elementary (but useful) characterization of the polar property.

Proposition 3. A subspace $Z$ of a dual space $X^{*}$ has the polar property if and only if for every linear function $f: \mathbb{R}^{k} \longrightarrow \mathbb{R}^{l}$, we have

$$
f^{*} X^{*}{ }^{-1}\left(B_{\ell_{\infty}^{k}\left(X^{*}\right)}\right)={\overline{\left.f^{*} X^{*}\right|_{\ell_{\infty}^{l}(Z)} ^{-1}\left(B_{\ell_{\infty}^{k}(Z)}\right)}}^{\sigma\left(\ell_{\infty}^{l}\left(X^{*}\right), \ell_{1}^{l}(X)\right)} .
$$

The following result shows that the polar property is a useful tool to study finite representability.

Theorem 4. A subspace $Z$ of a dual space $X^{*}$ has the polar property if and only if $X^{*}$ is finitely representable in $Z$ preserving the duality.

Proof. Assume that $X^{*}$ is f.d.-r. in $Z$ and $Z$ does not have the polar property. In virtue of Proposition 3 , there are $L \in \mathcal{L}\left(\ell_{1}^{k}(X), \ell_{1}^{l}(X)\right)$, a $0<\theta<1$ and a $l$-tuple

$$
\left(f_{i}\right)_{i=1}^{l} \in L^{*-1} B_{\ell_{\infty}^{k}\left(X^{*}\right)}{\overline{\left(\left.L^{*}\right|_{\ell_{\infty}^{l}(Z)} ^{-1} B_{\ell_{\infty}^{k}(Z)}\right)}}^{\sigma\left(\ell_{\infty}^{l}\left(X^{*}\right), \ell_{1}^{l}(X)\right)}
$$

such that $L^{*}\left(\left(f_{i}\right)_{i=1}^{l}\right)=\left(g_{i}\right)_{i=1}^{k} \in(1-\theta) B_{\ell_{\infty}^{k}\left(X^{*}\right)}$.

By the Hahn-Banach Theorem, there exists a number $0<\varepsilon<1$ and a $l$-tuple $\left(x_{i}\right)_{i=1}^{l} \in \ell_{1}^{l}(X)$ such that the $\sigma\left(\ell_{\infty}^{l}\left(X^{*}\right), \ell_{1}^{l}(X)\right)$-neighborhood of $\left(f_{i}\right)_{i=1}^{l}$, given by

$$
\mathcal{V}:=\left\{\left(h_{i}\right)_{i=1}^{l}:\left|\sum_{i=1}^{l}\left(f_{i}-h_{i}\right)\left(x_{i}\right)\right|<\varepsilon\right\}
$$


verifies

$$
\mathcal{V} \cap{\overline{L^{*}}}_{\left.\right|_{\ell_{\infty}^{l}(Z)} ^{-1} B_{\ell_{\infty}^{k}(Z)}}^{\sigma\left(\ell_{\infty}^{l}\left(X^{*}\right), \ell_{1}^{l}(X)\right)}=\emptyset .
$$

Since $X^{*}$ is f.d.-r. in $Z$, given $\delta>0$ such that $(1-\theta)(1+\delta) \leq 1$, there are $\tilde{f}_{1}, \ldots, \tilde{f}_{l}$ in $Z$ such that the operator

$$
G:\left\langle\tilde{f}_{i}: i=1, \ldots, l\right\rangle \longrightarrow\left\langle f_{i}: i=1, \ldots, l\right\rangle
$$

given by $G\left(\widetilde{f}_{i}\right):=f_{i}$ is a $\delta$-isometry and $\widetilde{f}_{i}\left(x_{j}\right)=f_{i}\left(x_{j}\right)$ for all $i$ and all $j$.

Associated to $L^{*}$, there is a matrix of numbers $\left(\lambda_{i j}\right)_{i=1 j=1}^{l}$ such that

$$
L^{*}\left(\left(h_{i}\right)_{i=1}^{l}\right)=\left(\sum_{i=1}^{l} \lambda_{i j} h_{i}\right)_{j=1}^{k}
$$

So, every $g_{j}$ is equal to $\sum_{i=1}^{l} \lambda_{i j} f_{i} \in(1-\theta) B_{X^{*}}$. Since $G$ is a $\delta$-isometry, for every $j=1, \ldots, k$, we have that $\widetilde{g}_{j}:=\sum_{i=1}^{l} \lambda_{i j} \widetilde{f}_{i} \in B_{Z}$, hence $\left(\widetilde{f}_{i}\right)_{i=1}^{l} \in$ $\left.L^{*}\right|_{\ell_{\infty}^{l}(Z)} ^{-1}\left(B_{\ell_{\infty}^{k}(Z)}\right)$. On the other hand, the equalities $\widetilde{f}_{i}\left(x_{j}\right)=f_{i}\left(x_{j}\right)$ for all $i=1, \ldots, l$ and all $j=1, \ldots, l$ imply that $\left(\widetilde{f}_{i}\right)_{i=1}^{l} \in \mathcal{V}$, in contradiction with (1).

For the converse implication, let $E$ and $F$ be finite dimensional subspaces of $X^{*}$ and $X$, let us denote $n:=\operatorname{dim} E$ and $k:=\operatorname{dim} F$, and let $0<\varepsilon<1$.

The Auerbach Lemma allows us to take $\left(h_{j}, y_{j}\right)_{j=1}^{n}$ in $E^{*} \times E$ such that $\left\|h_{j}\right\|=\left\|y_{j}\right\|=1$ for all $j=1, \ldots, n$ and $h_{i}\left(y_{j}\right)=\delta_{i j}$. The identity $i d$ : $E \longrightarrow X^{*}$ is given by $i d(e)=\sum_{j=1}^{n} h_{j}(e) y_{j}$. We shall find $z_{1}, \ldots, z_{n}$ in $Z$ for the wished $\varepsilon$-isometry $L: E \longrightarrow Z$ to be defined as $L(e):=\sum_{j=1}^{n} h_{j}(e) z_{j}$.

Let us take $0<\alpha<\min \left\{2 / 5,(1-\varepsilon)^{-1}-1, \varepsilon(1+n / 2)^{-1}\right\}$ and choose

$$
\begin{aligned}
& \text { a basis }\left\{x_{j}\right\}_{j=1}^{k} \text { in } F, \\
& \text { an } \alpha / 4 \text {-net }\left\{e_{j}\right\}_{j=1}^{N} \text { in } B_{E}, \text { and } \\
& \text { vectors }\left\{u_{j}\right\}_{j=1}^{N} \text { in } B_{X}
\end{aligned}
$$

such that $\|e\| \leq(1+\alpha) \sup _{1 \leq j \leq N}\left|e\left(u_{j}\right)\right|$ for all $e \in E$. The condition $\alpha<2 / 5$ guarantees the existence of the vectors $u_{j}$. We write

$$
e_{j}=\sum_{s=1}^{n} \lambda_{s}^{j} y_{s}, j=1, \ldots, N
$$


In order to simplify the notation, we denote

$$
\begin{aligned}
& U:=\ell_{1}^{n}(X), \\
& M:=U \oplus_{1} \ell_{1}^{N}(X), \\
& W:=\ell_{\infty}^{n}(Z), \\
& V:=W \oplus_{\infty} \ell_{\infty}^{N}(Z) .
\end{aligned}
$$

Let $\Lambda^{*}: U^{*} \longrightarrow M^{*}=U^{*} \oplus_{\infty} \ell_{\infty}^{N}\left(X^{*}\right)$ and $S^{*}: U^{*} \longrightarrow \mathbb{R}^{n k \times n N}$ be the conjugate operators given respectively by

$$
\Lambda^{*}\left(\left(g_{s}\right)_{s=1}^{n}\right):=\left(\left(g_{s}\right)_{s=1}^{n},\left(\sum_{s=1}^{n} \lambda_{s}^{j} g_{s}\right)_{j=1}^{N}\right)
$$

and

$$
S^{*}\left(\left(g_{s}\right)_{s=1}^{n}\right):=\left(g_{r}\left(x_{i}\right), g_{t}\left(u_{j}\right)\right) .
$$

Since $Z$ has the polar property, Proposition 3 gives that

$$
\Lambda^{*-1}\left(B_{M^{*}}\right)={\overline{\left.\Lambda^{*}\right|_{W} ^{-1}\left(B_{V}\right)}}^{\sigma\left(U^{*}, U\right)} .
$$

So, the $\sigma\left(U^{*}, U\right)$-continuity of $S^{*}$ yields

$$
S^{*}\left(\Lambda^{*-1}\left(B_{M^{*}}\right)\right) \subset \overline{S^{*}\left(\left.\Lambda^{*}\right|_{W} ^{-1}\left(B_{V}\right)\right)} .
$$

Observe that $R\left(S^{*}\right)=R\left(\left.S^{*}\right|_{W}\right)$ because $\bar{W}^{\sigma\left(U^{*}, U\right)}=U^{*}$. Thus, by Formula 2, since $\left(y_{j}\right)_{j=1}^{n} \in \Lambda^{*-1}\left(B_{M^{*}}\right)$, given any number $\varepsilon^{\prime}$ such that $0<$ $\varepsilon^{\prime}<\alpha\left\|\Lambda^{*}\right\|^{-1}$, we can find $\left.\left(c_{j}\right)_{j=1}^{n} \in \Lambda^{*}\right|_{W} ^{-1}\left(B_{V}\right)$ and $\left(b_{j}\right)_{j=1}^{n} \in \varepsilon^{\prime} B_{W}$ so that

$$
S^{*}\left(\left(y_{j}\right)_{j=1}^{n}\right)=S^{*}\left(\left(c_{j}\right)_{j=1}^{n}\right)+S^{*}\left(\left(b_{j}\right)_{j=1}^{n}\right) .
$$

Taking $z_{j}:=c_{j}+b_{j}$ for each $j=1, \ldots, n$ in the definition of the operator $L$, we obtain $\left(z_{j}\right)_{j=1}^{n} \in(1+\alpha) \Lambda^{*-1}\left(B_{V}\right)$ and $S^{*}\left(\left(y_{j}\right)_{j=1}^{n}\right)=S^{*}\left(\left(z_{j}\right)_{j=1}^{n}\right)$. Evidently, the condition $(L e)(x)=e(x)$ holds for all $e \in E$ and all $x \in F$.

In order to check that $L$ is an $\varepsilon$-isometry, let $e \in B_{E}$. On the one hand, we have

$$
\|L e\| \geq \sup _{1 \leq j \leq N}\left|(L e)\left(u_{j}\right)\right|=\sup _{1 \leq j \leq N}\left|e\left(u_{j}\right)\right| \geq(1+\alpha)^{-1}\|e\| \geq 1-\varepsilon .
$$

On the other hand, first note that $\|L\| \leq 2 n$ because

$$
\|L e\|=\left\|\sum_{j=1}^{n} h_{j}(e) z_{j}\right\| \leq \sum_{j=1}^{n}(1+\alpha)\left\|h_{j}\right\| \leq 2 n .
$$

Choose now vectors $e_{k}$ so that $\left\|e-e_{k}\right\| \leq \alpha / 4$. Since $\Lambda^{*}\left(\left(z_{j}\right)_{j=1}^{n}\right) \in(1+\alpha) B_{V}$ we have that $\left\|L e_{k}\right\|=\left\|\sum_{s=1}^{n} \lambda_{s}^{k} z_{s}\right\| \leq 1+\alpha$. Therefore,

$$
\|L e\| \leq\left\|L e_{k}\right\|+\left\|L\left(e-e_{k}\right)\right\| \leq 1+\varepsilon
$$

hence $L$ is an $\varepsilon$-isometry. 
Remark. If we take $f=i d: \mathbb{R} \longrightarrow \mathbb{R}$ in Proposition 3 , then we obtain that $B_{X^{*}}=\bar{B}_{Z}{ }^{\sigma\left(X^{*}, X\right)}$. Thus, if a subspace $Z$ of a dual space $X$ has the polar property, then $Z$ is norming on $X[\mathbf{1}]$.

The converse implication is not true. There are norming subspaces of dual Banach spaces which fail the polar property.

For example, let us consider the Rademacher-like sequence $\left(x_{n}\right)$ in $\ell_{\infty}$, where $x_{1}=(1,-1,1,-1,1,-1, \ldots)$ and for $n \in \mathbb{N}$ the sequence $x_{n+1}$ consists of successive repetitions of the block

$$
1, \stackrel{\left(2^{n}\right)}{\cdot}, 1,-1, \stackrel{\left(2^{n}\right)}{\cdot},-1 \text {. }
$$

Then $\left(x_{n}\right)$ is 1 -equivalent to the unit vector basis of $\ell_{1}$. Now, if we take an enumeration $\left\{A_{n}: n \in \mathbb{N}\right\}$ of all the finite sequences of numbers in $\{1,-1\}$, with $\operatorname{card}\left(A_{m}\right) \leq \operatorname{card}\left(A_{n}\right)$ for $m<n$, and modify each $x_{n}$ in a finite number of coordinates so that the initial segment of $x_{n}$ coincides with $A_{n}$, then $\left(x_{n}\right)$ continues to be 1-equivalent to the unit vector basis of $\ell_{1}$, and it generates a norming subspace $\left[x_{n}\right]$ of $\ell_{\infty}=\ell_{1}^{*}$.

However, since $\ell_{\infty}$ is not f.r. in $\ell_{1}$, the subspace $\left\langle x_{n}\right\rangle$ of $\ell_{\infty}$ does not have the polar property, by Theorem 4 .

The following concept is a generalization of the polar property.

Definition 5. Let $\mathcal{A}$ be a class of operators. A subspace $Z$ of $X^{*}$ is said to have the $\mathcal{A}$-polar property if given a Banach $Y$, an operator $T \in \mathcal{A}(Y, X)$, integers $p, q \in \mathbb{N}$ and $r \in \mathbb{N} \cup\{0\}$, and a couple of matrices of real numbers $\left(a_{i j}\right)_{i=1 j=1}^{q},\left(b_{i j}\right)_{i=1 j=1}^{r}$, we have that the operator $L: \ell_{1}^{q}(X) \oplus_{1} \ell_{1}^{r}(Y) \longrightarrow$ $\ell_{1}^{p}(X)$, given by

$$
L\left(\left(x_{i}\right)_{i=1}^{q},\left(y_{j}\right)_{j=1}^{r}\right):=\left(\sum_{i=1}^{q} a_{i j} x_{i}+\sum_{i=1}^{r} b_{i j} T y_{i}\right)_{j=1}^{p},
$$

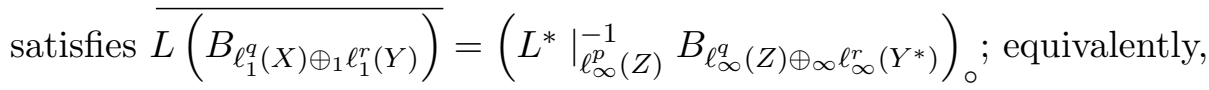

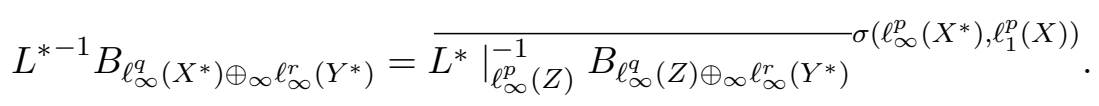

Remark. In the case $r=0$, we assume that the matrix $\left(b_{i j}\right)_{i=1 j=1}^{r} \underset{j}{p}$ appearing in Definition 5 has no entries. Therefore, the $\mathcal{A}$-polar property implies the polar property.

The polar property is equivalent to the $\mathcal{I}$-polar property, where $\mathcal{I}$ stands for the class of all identities of Banach spaces.

The difficulty to check the $\mathcal{A}$-polar property depends on the structure of $\mathcal{A}$. Sometimes it is possible to do it easily, as in the next example which will play an important role in Proposition 13. 
Example 6. Let $\mathcal{B}^{d}:=\left\{T^{*}: T \in \mathcal{B}\right\}$ denote the class of all conjugate operators. Then every Banach space $X$ has the $\mathcal{B}^{d}$-polar property as a subspace of $X^{* *}$.

In fact, take an operator $T^{*}: Y^{*} \longrightarrow X^{*}$, and matrices $\left(a_{i j}\right)_{i=1 j=1}^{q} \underset{\text {, }}{p}$, $\left(b_{i j}\right)_{i=1 j=1}^{r}$ of real numbers. As in Definition 5, we define $L: \ell_{1}^{q}\left(X^{*}\right) \oplus_{1}$ $\ell_{1}^{r}\left(Y^{*}\right) \longrightarrow \ell_{1}^{p}\left(X^{*}\right)$ by

$$
L\left(\left(f_{i}\right)_{i=1}^{q},\left(g_{j}\right)_{j=1}^{r}\right):=\left(\sum_{i=1}^{q} a_{i j} f_{i}+\sum_{i=1}^{r} b_{i j} T^{*} g_{i}\right)_{j=1}^{p} .
$$

Let us write $U:=\ell_{\infty}^{q}(X) \oplus_{\infty} \ell_{\infty}^{r}(Y)$ and $V:=\ell_{\infty}^{p}(X)$. Clearly, $L$ can be identified with a conjugate operator $S^{*}: U^{*} \longrightarrow V^{*}$, and consequently, we have that $S^{*}\left(B_{U^{*}}\right)=\left(S^{-1} B_{U}\right)_{\circ}$; therefore,

$$
S^{*}\left(B_{U^{*}}\right)=\left(\left.S^{* *}\right|_{V} ^{-1} B_{\ell_{\infty}^{q}(X) \oplus \infty \ell_{\infty}^{r}\left(Y^{* *}\right)}\right)_{\circ} .
$$

We saw in Theorem 4 that a subspace $Z$ of $X^{*}$ has the polar property if and only if $X^{*}$ is f.d.-r. in $Z$. Now we prove a strengthening of the direct implication.

Theorem 7. Let $Z$ be a subspace of $X^{*}$, let $E$ and $F$ be finite dimensional subspaces of $X^{*}$ and $X$ respectively, and let $\mathcal{A}$ be a class of operators.

If $Z$ has the $\mathcal{A}$-polar property and $T \in \mathcal{A}(Y, X)$, then for every $0<\varepsilon<1$ and $\delta^{\prime}>0$ there is an $\varepsilon$-isometry $L: E \longrightarrow Z$ such that $(L e)(x)=e(x)$ for all $e \in E$ and all $x \in F$, and satisfies the following additional condition $\left\|\left.T^{*}\right|_{L(E)}\right\| \leq\left\|\left.T^{*}\right|_{E}\right\|+\delta^{\prime}$.

Proof. It is similar to the second part of the proof of Theorem 4. We shall use here the same notations.

Let $\delta:=\|T\|$. Without loss of generality, we suppose that $0<\varepsilon<$ $2^{-3} \delta^{-1} \delta^{\prime}$. We choose $0<\alpha<\min \left\{2 / 5,(1-\varepsilon)^{-1}-1, \varepsilon(1+n / 2)^{-1}\right\}$ and define the operator $\Lambda^{*}$ as in the proof of Theorem 4. Moreover, we write $\widetilde{M}^{*}:=M^{*} \oplus_{\infty} \ell_{\infty}^{N}\left(Y^{*}\right)$, and define the conjugate operator $\widetilde{\Lambda}^{*}: U^{*} \longrightarrow \widetilde{M}^{*}$ by

$$
\widetilde{\Lambda}^{*}\left(\left(g_{s}\right)_{s=1}^{n}\right):=\left(\Lambda^{*}\left(\left(g_{s}\right)_{s=1}^{n}\right),\left(\delta^{-1} T^{*}\left(\sum_{s=1}^{n} \lambda_{s}^{j} g_{s}\right)\right)_{j=1}^{N}\right) .
$$

Write $\widetilde{V}:=V \oplus_{\infty} \ell_{\infty}^{N}\left(Y^{*}\right)$. Since $Z$ has the $\mathcal{A}$-polar property, we have

$$
{\overline{\widetilde{\Lambda}^{*}}}_{\left.\right|_{W} ^{-1}\left(B_{\widetilde{V}}\right)}^{\sigma\left(U^{*}, U\right)}=\widetilde{\Lambda}^{*-1}\left(B_{\widetilde{M}^{*}}\right) .
$$

Thus, $\left(y_{j}\right)_{j=1}^{n} \in \widetilde{\Lambda}^{*-1}\left(B_{\widetilde{M}^{*}}\right)$, and proceeding as in the proof of Theorem 4 , we find

$$
\left.\left(z_{j}\right)_{j=1}^{n} \in(1+\alpha) \widetilde{\Lambda}^{*}\right|_{W} ^{-1}\left(B_{\widetilde{V}}\right)
$$


such that $S^{*}\left(\left(y_{j}\right)_{j=1}^{n}\right)=S^{*}\left(\left(z_{j}\right)_{j=1}^{n}\right)$. Again as in the proof of Theorem 4 , the condition $\alpha<\min \left\{2 / 5,(1-\varepsilon)^{-1}-1, \varepsilon(1+n / 2)^{-1}\right\}$ implies that the operator $L: E \longrightarrow Z$ is an $\varepsilon$-isometry and $(L e)(x)=e(x)$ for $e \in E$ and $x \in F$. Let us check that $\left\|\left.T^{*}\right|_{L(E)}\right\| \leq \delta+\delta^{\prime}$. Note that $\left(z_{s}\right)_{s=1}^{n} \in(1+\alpha) \widetilde{\Lambda}^{*-1}\left(B_{\widetilde{V}}\right)$ implies that

$$
\left\|T^{*}\left(L e_{j}\right)\right\|=\left\|T^{*}\left(\sum_{s=1}^{n} \lambda_{s}^{j} z_{s}\right)\right\| \leq \delta(1+\alpha) \text { for } j=1, \ldots, N .
$$

Since $L$ is an $\varepsilon$-isometry, if $\beta:=4^{-1} \alpha(1+\varepsilon)+2 \varepsilon$, then $\left\{L e_{j}\right\}_{j=1}^{N}$ is a $\beta$-net in $(1+\varepsilon) B_{L(E)}$, and taking $w_{j}:=(1+\varepsilon)^{-1} L e_{j}$ we obtain a $(1+\varepsilon)^{-1} \beta$ net $\left\{w_{j}\right\}_{j=1}^{N}$ in $B_{L(E)}$. Let $w \in B_{L(E)}$ and pick $w_{j}$ such that $\left\|w-w_{j}\right\| \leq$ $(1+\varepsilon)^{-1} \beta$. We have

$$
\left\|T^{*} w\right\| \leq\left\|T^{*}\left(w-w_{j}\right)\right\|+\left\|T^{*} w_{j}\right\| \leq \frac{\beta}{1+\varepsilon}\left\|T^{*}\right\|+\frac{\delta(1+\alpha)}{1+\varepsilon} .
$$

Since $\alpha<\left\{2 / 5,2^{-2} \delta^{-1} \delta^{\prime}\right\}$ and $\varepsilon<2^{-3} \delta^{-1} \delta^{\prime}$, we conclude $\left\|T^{*} w\right\| \leq \delta+$ $\delta^{\prime}$.

As a consequence of Theorem 7, we derive a version of the principle of local reflexivity [8].

Corollary 8. Given an operator $T \in \mathcal{B}(X, Y)$, a pair of finite dimensional subspaces $E \subset X^{* *}$ and $F \subset X^{*}$, and numbers $0<\varepsilon<1$ and $\delta>0$, there exists an $\varepsilon$-isometry $L: E \longrightarrow X$ such that we have $f(L e)=e(f)$ for $f \in F$ and $e \in E$, and $\left\|\left.T\right|_{L(E)}\right\|<\left\|\left.T^{* *}\right|_{E}\right\|+\delta$.

Proof. We have seen in Example 6 that $X$ has the $\mathcal{B}^{d}$-polar property as a subspace of $X^{* *}$. Thus, $X$ plays here the role of $Z$ in Theorem 7 as a subspace of the dual space $\left(X^{*}\right)^{*}$, and the result holds.

\section{Applications.}

Here we apply the previous results to study the duality theory for ultrapowers of operators and some semigroups of operators related with the superreflexivity and the finite representability of $c_{0}$ and $\ell_{1}$ in a Banach space.

First, we show in Theorem 10 that for every operator $T$, the kernel $\operatorname{ker}\left(T^{* *} \mathfrak{U}\right)$ is f.r. in $\operatorname{ker}\left(T_{\mathfrak{U}}\right)$. In order to do that, we need the following technical Lemma.

Lemma 9. Given an ultrafilter $\mathfrak{U}$ on $I$ and a sequence $\left(B_{n}\right) \subset \mathfrak{U}$ such that $B_{n+1} \subset B_{n}$ for every $n$, there is a sequence $\left(C_{n}\right) \subset \mathfrak{U}$ verifying $C_{n+1} \subset$ $C_{n} \subset B_{n}, C_{n} \backslash C_{n+1} \neq \emptyset$ for every $n \in \mathbb{N}$, and $\bigcap_{n=1}^{\infty} C_{n}=\emptyset$. 
Proof. Let $\left\{I_{n}: n \in \mathbb{N}\right\}$ be a partition of $I$ such that $\emptyset \neq I_{n} \notin \mathfrak{U}$ for every $n \in \mathbb{N}$. We build the sets $C_{n}$ inductively. We take $C_{1}:=B_{1}$. Assume we already have the sets $\left(C_{n}\right)_{n=1}^{k} \subset \mathfrak{U}$ verifying $C_{n+1} \subset C_{n} \subset B_{n}$ and $C_{n+1} \neq$ $C_{n}$ for $n=1, \ldots, k-1$. Let us denote $n_{k}:=\min \left\{n \geq k: I_{n} \cap C_{k} \neq \emptyset\right\}$ and

$$
C_{k+1}:=\bigcup_{n=n_{k}+1}^{\infty}\left(I_{n} \cap B_{k+1}\right) \text {. }
$$

It is easy to check that the sequence $\left(C_{n}\right)_{n}$ satisfies the desired conditions.

Theorem 10. Let $T \in \mathcal{B}(X, Y)$ be an operator and let $\mathfrak{U}$ be an ultrafilter on I. Then the kernel $\operatorname{ker}\left(T_{\mathfrak{U}}^{* *}\right)$ is finitely representable in $\operatorname{ker}\left(T_{\mathfrak{U}}\right)$.

Proof. Let $E$ be a finite dimensional subspace of $\operatorname{ker}\left(T^{* *} \mathfrak{U}\right)$ and let $\left\{\mathbf{F}_{k}=\right.$ $\left.\left[F_{k}^{i}\right]_{i}: k=1, \ldots, n\right\}$ be a basis of $E$. Let us denote $E_{i}:=\left\langle F_{k}^{i}: k=1, \ldots, n\right\rangle$ and let $T_{i}: E \longrightarrow E_{i}$ be the operator given by $T_{i}\left(\mathbf{F}_{k}\right):=F_{k}^{i}$.

Taking into account that $\lim _{\mathfrak{U}(i)} T^{* *} F_{k}^{i}=0$, a result of Heinrich [5, Proposition 6.1] allows us to find, for each positive integer $m$, a set $J_{m} \in \mathfrak{U}$ such that for every $i \in J_{m}$, the operators $T_{i}$ are $1 / m$-isometries and $\left\|T^{* *} F_{k}^{i}\right\|<$ $1 / m$ for $k=1, \ldots, n$. By Lemma 9 , we can take a decreasing sequence $\left(C_{n}\right)_{n}$ in $\mathfrak{U}$ such that $\cap_{n=1}^{\infty} C_{n}=\emptyset, C_{n} \neq C_{n+1}$ and $C_{n} \subset J_{n}$ for all $n \in \mathbb{N}$; add the set $C_{0}:=I$ to this sequence. For every $i \in I$, we denote by $m_{i}$ the unique element of $\mathbb{N} \cup\{0\}$ so that $i \in C_{m_{i}} \backslash C_{m_{i}+1}$.

For every $i \in C_{1}$, since $i \in C_{m_{i}} \backslash C_{m_{i}+1}$, by Corollary 8 we can choose $x_{1}^{i}, \ldots, x_{n}^{i}$ in $X$ such that the operator $L_{i}: E_{i} \longrightarrow F_{i}:=\left\langle x_{1}^{i}, \ldots, x_{n}^{i}\right\rangle$ given by $L_{i}\left(F_{k}^{i}\right):=x_{k}^{i}$ is a $1 / m_{i}$-isometry and $\left\|T x_{k}^{i}\right\| \leq 2 / m_{i}$ for $k=1, \ldots, n$.

Define $\mathbf{x}_{k}:=\left[x_{k}^{i}\right]$ and write $F:=\left\langle\mathbf{x}_{k}: k=1, \ldots, n\right\rangle$. Thus we have that every $\mathbf{x}_{k}$ belongs to $\operatorname{ker}\left(T_{\mathfrak{U}}\right)$ and $F$ is isometric to $E$. In fact, on the one hand $C_{m}=\left\{i: m_{i} \geq m\right\} \in \mathfrak{U}$ for each $m \in \mathbb{N}$. So $\left\|T x_{k}^{i}\right\| \leq 2 / m$ for all $i \in C_{m}$. Therefore $\lim _{\mathfrak{U}(i)} T x_{k}^{i}=0$ and $\mathbf{x}_{k} \in \operatorname{ker}\left(T_{\mathfrak{U}}\right)$ for $k=1, \ldots, n$.

On the other hand, let $\sum_{k=1}^{n} \lambda_{k} \mathbf{F}_{k}$ be a norm-one element in $E$. Let $M$ be an upper bound for $\left\{\sum_{k=1}^{n} \lambda_{k} F_{k}^{i}: i \in I\right\}$. Take any $m \in \mathbb{N}$. For every $i \in\left\{i: m_{i} \geq m\right\}$, we have that $L_{i}$ is a $1 / m$-isometry; thus

$$
C_{m} \subset\left\{i:\left\|\sum_{k=1}^{n} \lambda_{k} F_{k}^{i}\right\|-\left\|\sum_{k=1}^{n} \lambda_{k} x_{k}^{i}\right\| \mid \leq M / m\right\} \in \mathfrak{U} .
$$

In this way we obtain that $\lim _{\mathfrak{U}(i)}\left\|\sum_{k=1}^{n} \lambda_{k} F_{k}^{i}\right\|-\left\|\sum_{k=1}^{n} \lambda_{k} x_{k}^{i}\right\|=0$. Since

$$
\left\|\sum_{k=1}^{n} \lambda_{k} \mathbf{x}_{k}\right\|=\lim _{\mathfrak{U}(i)}\left\|\sum_{k=1}^{n} \lambda_{k} x_{k}^{i}\right\| \text { and } \lim _{\mathfrak{U}(i)}\left\|\sum_{k=1}^{n} \lambda_{k} F_{k}^{i}\right\|=\left\|\sum_{k=1}^{n} \mathbf{F}_{k}\right\|,
$$

we get $\left\|\sum_{k=1}^{n} \lambda_{k} \mathbf{x}_{k}\right\|=\left\|\sum_{k=1}^{n} \mathbf{F}_{k}\right\|$. Hence, $E$ is isometric to $F$ and therefore $\operatorname{ker}\left(T^{* *} \mathfrak{U}\right)$ is f.r. in $\operatorname{ker}\left(T_{\mathfrak{U}}\right)$. 
A second application of Theorem 4: Heinrich [5] showed that $X_{\mathfrak{U}}{ }^{*}$ is f.d.-r. in $X^{*} \mathfrak{U}$. This is a consequence of our Theorem 11 below, applied to the zero operator. But our Theorem 4 is stronger than the result in [5]. Indeed, it will allow us to prove in Theorem 11 that $\operatorname{ker}\left(T_{\mathfrak{U}}{ }^{*}\right)$ is f.d.-r. in $\operatorname{ker}\left(T^{*} \mathfrak{U}\right)$ for any operator $T \in \mathcal{B}(X, Y)$. Note that $\operatorname{ker}\left(T_{\mathfrak{U}}{ }^{*}\right)$ can be identified with the dual space $\operatorname{coker}\left(T_{\mathfrak{U}}\right)^{*}$, but is not necessarily the second dual of any space. Moreover, in general coker $\left(T_{\mathfrak{U}}\right)$ is not an ultrapower [3]. Thus neither the principle of local reflexivity nor the result of Heinrich are applicable.

Theorem 11. Let $T: X \longrightarrow Y$ be an operator and $\mathfrak{U}$ and ultrafilter on $I$. Then the kernel $\operatorname{ker}\left(T_{\mathfrak{U}}{ }^{*}\right)$ is finitely representable in $\operatorname{ker}\left(T^{*} \mathfrak{U}\right)$ preserving the duality.

Proof. By Theorem 4, it is enough to prove that $\operatorname{ker}\left(T^{*} \mathfrak{U}\right)$ has the polar property as a subspace of $\operatorname{ker}\left(T_{\mathfrak{U}}{ }^{*}\right)$. We identify isometrically the kernel $\operatorname{ker}\left(T_{\mathfrak{U}}{ }^{*}\right)$ with the dual of the cokernel $\operatorname{coker}\left(T_{\mathfrak{U}}\right)$. Along this proof, when we say that an operator $A: U_{1} \longrightarrow U_{2}$ is identified with $B: V_{1} \longrightarrow V_{2}$ we mean that there are isometries onto $J_{1}: U_{1} \longrightarrow V_{1}$ and $J_{2}: U_{2} \longrightarrow V_{2}$ so that $A=J_{2}^{-1} B J_{1}$.

Pick a linear function $f: \mathbb{R}^{k} \longrightarrow \mathbb{R}^{l}$ and write

$$
f_{\text {coker }\left(T_{\mathfrak{U}}\right)}: \ell_{1}^{k}\left(\operatorname{coker}\left(T_{\mathfrak{U}}\right)\right) \longrightarrow \ell_{1}^{l}\left(\operatorname{coker}\left(T_{\mathfrak{U}}\right)\right) .
$$

We only have to check the inclusion

$$
\left(\left.\left(f_{\text {coker }\left(T_{\mathfrak{U}}\right)}\right)^{*}\right|_{\ell_{\infty}^{l}\left(\operatorname{ker}\left(T^{*} \mathfrak{U}\right)\right)} ^{-1} B_{\ell_{\infty}^{k}\left(\operatorname{ker}\left(T^{*} \mathfrak{U}\right)\right)}\right)_{\circ} \subset \overline{f_{\operatorname{coker}\left(T_{\mathfrak{U}}\right)} B_{\ell_{1}^{k}\left(\operatorname{coker}\left(T_{\mathfrak{U}}\right)\right)}} .
$$

The proof is divided into three cases. The main one is the case $k=l$. The cases $k<l$ and $k>l$ will be obtained as a consequence of the main case.

Case a) $k=l$. We consider the operator $T^{k}: \ell_{1}^{k}(X) \longrightarrow \ell_{1}^{k}(Y)$, defined by $T^{k}\left(\left(x_{j}\right)_{j=1}^{k}\right):=\left(T x_{j}\right)_{j=1}^{k}$, and write $U:=T^{k}, V:=\ell_{1}^{k}(X)$ and $W:=\ell_{1}^{k}(Y)$. We can identify $U_{\mathfrak{U}}: V_{\mathfrak{U}} \longrightarrow W_{\mathfrak{U}}$ with

$$
\left(T_{\mathfrak{U}}\right)^{k}: \ell_{1}^{k}\left(X_{\mathfrak{U}}\right) \longrightarrow \ell_{1}^{k}\left(Y_{\mathfrak{U}}\right) \text {. }
$$

The operator $\varphi: W_{\mathfrak{U}} \longrightarrow \ell_{1}^{k}\left(\operatorname{coker}\left(T_{\mathfrak{U}}\right)\right)$ which sends $\left[\left(y_{i}^{j}\right)_{j=1}^{k}\right]_{i}$ to $\left(\left[y_{i}^{j}\right]_{i}+\right.$ $\left.\left.\overline{R\left(T_{\mathfrak{U}}\right.}\right)\right)_{j=1}^{k}$ is surjective, $\operatorname{ker}(\varphi)=\overline{R\left(U_{\mathfrak{U}}\right)}$ and it is easy to check that the induced operator

$$
\widetilde{\varphi}: \operatorname{coker}\left(U_{\mathfrak{U}}\right) \longrightarrow \ell_{1}^{k}\left(\operatorname{coker}\left(T_{\mathfrak{U}}\right)\right)
$$

is an isometry. Now, since $\left(f_{Y}\right)_{\mathfrak{U}}: W_{\mathfrak{U}} \longrightarrow W_{\mathfrak{U}}$ satisfies $\left(f_{Y}\right)_{\mathfrak{U}}\left(R\left(U_{\mathfrak{U}}\right)\right) \subset$ $R\left(U_{\mathfrak{U}}\right)$, it induces an operator $L: \operatorname{coker}\left(U_{\mathfrak{U}}\right) \rightarrow \operatorname{coker}\left(U_{\mathfrak{U}}\right)$ given by

$$
L\left(\left[y_{i}\right]+\overline{R\left(U_{\mathfrak{U}}\right)}\right):=\left(f_{Y}\right)_{\mathfrak{U}}\left(\left[y_{i}\right]\right)+\overline{R\left(U_{\mathfrak{U}}\right)} .
$$

The operator $L$ is identified with

$$
f_{\operatorname{coker}\left(T_{\mathfrak{U}}\right)}: \ell_{1}^{k}\left(\operatorname{coker}\left(T_{\mathfrak{U}}\right)\right) \longrightarrow \ell_{1}^{k}\left(\operatorname{coker}\left(T_{\mathfrak{U}}\right)\right)
$$


because $L=\widetilde{\varphi}^{-1} f_{\operatorname{coker}\left(T_{\mathfrak{U}}\right)} \widetilde{\varphi}$. Since $\ell_{1}^{k}\left(\operatorname{coker}\left(T_{\mathfrak{U}}\right)\right)^{*}=\ell_{\infty}^{k}\left(\operatorname{ker}\left(T_{\mathfrak{U}}{ }^{*}\right)\right)$, we can identify

$$
\left(f_{\operatorname{coker}\left(T_{\mathfrak{U}}\right)}\right)^{*}: \ell_{\infty}^{k}\left(\operatorname{ker}\left(T_{\mathfrak{U}}{ }^{*}\right)\right) \rightarrow \ell_{\infty}^{k}\left(\operatorname{ker}\left(T_{\mathfrak{U}}{ }^{*}\right)\right)
$$

with $L^{*}: \operatorname{ker}\left(U_{\mathfrak{U}}{ }^{*}\right) \longrightarrow \operatorname{ker}\left(U_{\mathfrak{U}}{ }^{*}\right)$. Under the previous identification we have that $\operatorname{ker}\left(U^{*} \mathfrak{U}\right)=\ell_{\infty}^{k}\left(\operatorname{ker}\left(T^{*} \mathfrak{U}\right)\right)$. So the result that we want to prove (Formula 3$)$ is equivalent to the inclusion

$$
\left(\left.L^{*}\right|_{\operatorname{ker}\left(U^{*} \mathfrak{U}\right)} ^{-1} B_{\operatorname{ker}\left(U^{*} \mathfrak{U}\right)}\right)_{\circ} \subset \overline{L B_{\operatorname{coker}\left(U_{\mathfrak{U}}\right)}} .
$$

Suppose that this inclusion is false. Then there exists $\mathbf{h}=\left[h_{i}\right] \in W_{\mathfrak{U}}$ such that

$$
\mathbf{h}+\overline{R\left(U_{\mathfrak{U}}\right)} \in\left(L^{*-1} B_{\operatorname{ker}\left(U^{*} \mathfrak{U}\right)}\right)_{\diamond} \backslash \overline{L B_{\operatorname{coker}\left(U_{\mathfrak{U}}\right)}} .
$$

We can assume that $\left\|h_{i}\right\|=K>0$ for all $i$. Write $S:=f_{Y}, A:=S\left(B_{W}\right)$ and $\mathbf{A}:=S_{\mathfrak{U}}\left(B_{W_{\mathfrak{U}}}\right)=\left\{\left[a_{i}\right]: a_{i} \in A, i \in I\right\}$. Take $\varepsilon>0$ such that

$$
\operatorname{dist}\left(\mathbf{h}-\mathbf{A}, R\left(U_{\mathfrak{U}}\right)\right)>\varepsilon>0 \text {. }
$$

Thus, for every $m \in \mathbb{N}$, we have $\operatorname{dist}\left(\mathbf{h}-\mathbf{A}, m U_{\mathfrak{U}} B_{V_{\mathfrak{U}}}\right)>\varepsilon$, hence

$$
D_{m}:=\left\{i \in I: \operatorname{dist}\left(h_{i}-A, m U B_{V}\right)>\varepsilon\right\} \in \mathfrak{U} .
$$

By Lemma 9 we can take a sequence $\left(C_{n}\right)_{n}$ in $\mathfrak{U}$ such that $C_{n+1} \subset C_{n} \subset D_{n}$ and $C_{n} \neq C_{n+1}$ for every $n \in \mathbb{N}$, and $\cap_{n=1}^{\infty} C_{n}=\emptyset$.

For every $i \in I$, let $m_{i}$ be the unique positive integer for which $i \in$ $C_{m_{i}} \backslash C_{m_{i}+1}$, and denote $K_{i}:=h_{i}-A-m_{i} U B_{V}$. Since $K_{i}$ is convex and $K_{i} \cap \varepsilon B_{W}=\emptyset$, by the Hahn-Banach Theorem there is a vector $f_{i} \in S_{W^{*}}$ such that inf $f_{i}\left(K_{i}\right) \geq \varepsilon$. Hence

$$
\left|f_{i}(U x)\right| \leq \frac{K+\|S\|+\varepsilon}{m_{i}} \quad \text { for all } x \in B_{V} ;
$$

equivalently, $\left\|U^{*} f_{i}\right\| \leq m_{i}{ }^{-1}(K+\|S\|+\varepsilon)$. Since the chain $\left(C_{n}\right)_{n}$ has empty intersection we have $\lim _{\mathfrak{U}} m_{i}{ }^{-1}=0$, and therefore $\mathbf{f}:=\left[f_{i}\right] \in \operatorname{ker}\left(U^{*} \mathfrak{U}\right)$.

On the other hand, inf $f_{i}\left(K_{i}\right) \geq \varepsilon \operatorname{implies} \inf f_{i}\left(h_{i}-A\right) \geq \varepsilon$. Thus we get $0<\varepsilon \leq \varepsilon_{i}:=f_{i}\left(h_{i}\right) \leq K$ for all $i$ and $\left|f_{i}(a)\right| \leq \varepsilon_{i}-\varepsilon$ for all $a \in A$. Hence, letting $g_{i}:=\varepsilon_{i}^{-1} f_{i}$, we obtain that

$$
\alpha:=\sup \left[g_{i}\right]\left(L B_{\operatorname{coker}\left(U_{\mathfrak{U}}\right)}\right) \leq 1-\varepsilon K^{-1}<1=\left[g_{i}\right]\left(\left[h_{i}\right]\right) .
$$

Take $y_{i}:=\alpha^{-1} g_{i}$. We have that $\mathbf{y}:=\left[y_{i}\right]=\alpha^{-1}\left(\lim _{\mathfrak{U}} \varepsilon_{i}^{-1}\right)\left[f_{i}\right] \in \operatorname{ker}\left(U^{*} \mathfrak{U}\right)$ and

$$
\sup \mathbf{y}\left(L B_{\operatorname{coker}\left(U_{\mathfrak{U}}\right)}\right) \leq 1<\mathbf{y}(\mathbf{h}) ;
$$

thus $\mathbf{y} \in L^{*-1} B_{\operatorname{ker}\left(U_{\mathfrak{U}}^{*}\right)}$ and $\mathbf{h}+\overline{R\left(U_{\mathfrak{U}}\right)} \notin\left(L^{*-1} B_{\operatorname{ker}\left(U^{*} \mathfrak{U}\right)}\right)_{\circ} \backslash \overline{L B_{\operatorname{coker}\left(U_{\mathfrak{U}}\right)}}$, a contradiction.

For the cases b) and c), we adopt the notations $Z:=\operatorname{coker}\left(T_{\mathfrak{U}}\right)$ and $H:=\operatorname{ker}\left(T^{*} \mathfrak{U}\right)$, so that $Z^{*}=\operatorname{ker}\left(T_{\mathfrak{U}}{ }^{*}\right)$. 
Case b) $k<l$. We consider the operator $\widetilde{L}: \ell_{1}^{k}(Z) \oplus_{1} \ell_{1}^{l-k}(Z) \longrightarrow \ell_{1}^{l}(Z)$, defined by $\widetilde{L}(a, b):=L a$. By case a) we have

$$
\widetilde{L} B_{\ell_{1}^{k}(Z) \oplus_{1} \ell_{1}^{l-k}(Z)}=\left(\left.\widetilde{L}^{*}\right|_{\ell_{\infty}^{l}(H)} ^{-1} B_{\ell_{\infty}^{k}(H) \oplus_{\infty} \ell_{\infty}^{l-k}(H)}\right)_{\circ} .
$$

Since $L B_{\ell_{1}^{k}(Z)}=\widetilde{L} B_{\ell_{1}^{k}(Z) \oplus_{1} \ell_{1}^{l-k}(Z)}$ and

$$
\left.L^{*}\right|_{\ell_{\infty}^{l}(H)} ^{-1}\left(B_{\ell_{\infty}^{k}(H)}\right)=\left.\widetilde{L}^{*}\right|_{\ell_{\infty}^{l}(H)} ^{-1}\left(B_{\ell_{\infty}^{k}(H) \oplus_{\infty} \ell_{\infty}^{l-k}(H)}\right),
$$

we have that $\overline{L B_{\ell_{1}^{k}(Z)}}=\left(\left.L^{*}\right|_{\ell_{\infty}^{l}(H)} ^{-1} B_{\ell_{\infty}^{k}(H)}\right)_{\circ}$.

Case c) $k>l$. We consider the operator $\widetilde{L}: \ell_{1}^{k}(Z) \longrightarrow \ell_{1}^{l}(Z) \oplus_{1} \ell_{1}^{k-l}(Z)$, defined by $\widetilde{L}(a):=(L a, 0)$. By case a) we have

$$
\widetilde{L} B_{\ell_{1}^{k}(Z)}=\left(\left.\widetilde{L}^{*}\right|_{\ell_{\infty}^{l}(H) \oplus_{\infty} \ell_{\infty}^{k-l}(H)} ^{-1} B_{\ell_{\infty}^{k}(H)}\right)_{\circ} .
$$

The definition of $\widetilde{L}$ yields $L\left(B_{\ell_{1}^{k}(Z)}\right) \times 0_{\ell_{1}^{k-l}(Z)}=\widetilde{L}\left(B_{\ell_{1}^{k}(Z)}\right)$ and

$$
\left.L^{*}\right|_{\ell_{\infty}^{l}(H)} ^{-1}\left(B_{\ell_{\infty}^{k}(H)}\right) \times \ell_{\infty}^{k-l}\left(Z^{*}\right)=\left.\widetilde{L}^{*}\right|_{\ell_{\infty}^{l}(H) \oplus_{\infty} \ell_{\infty}^{k-l}(H)} ^{-1}\left(B_{\ell_{\infty}^{k}(H)}\right) .
$$

So we obtain

$$
\left(\left.L^{*}\right|_{\ell_{\infty}^{l}(H)} ^{-1}\left(B_{\ell_{\infty}^{k}(H)}\right)\right)_{\circ} \times 0_{\ell_{1}^{k-l}(Z)}=\left(\left.\widetilde{L}^{*}\right|_{\ell_{\infty}^{l}(H) \oplus_{\infty} \ell_{\infty}^{k-l}(H)} ^{-1}\left(B_{\ell_{\infty}^{k}(H)}\right)\right)_{\circ} .
$$

Consequently, we get $\overline{L B_{\ell_{1}^{k}(Z)}}=\left(\left.L^{*}\right|_{\ell_{\infty}^{l}(H)} ^{-1}\left(B_{\ell_{\infty}^{k}(H)}\right)\right)_{\circ}$.

Corollary $12([\mathbf{9}])$. Let $T: X \longrightarrow Y$ be an operator and let $\mathfrak{U}$ be an ultrafilter on I. Then $\operatorname{ker}\left(T^{*} \mathfrak{U}\right)$ is a norming subspace of $\operatorname{ker}\left(T_{\mathfrak{U}}{ }^{*}\right)=\left(\operatorname{coker}\left(T_{\mathfrak{U}}\right)\right)^{*}$.

A class of operators $\mathcal{A}$ is said to be a semigroup if the composition of two operators of $\mathcal{A}$ belongs to $\mathcal{A}$ and if for every Banach space $X$, the identity operator $I_{X}$ on $X$ belongs to $\mathcal{A}$. As well-known examples of semigroups, we mention the classical semi-Fredholm operators, and the tauberian operators, introduced by Kalton and Wilansky [7]. An operator $T \in \mathcal{B}(X, Y)$ is tauberian if $T^{* *}\left(X^{* *} \backslash X\right) \subset Y^{* *} \backslash Y$.

Now we apply the previous results to study the dual properties of the semigroups $\mathcal{W}^{u p}{ }_{+}$, introduced in [12], and $\mathcal{U}^{u p}{ }_{+}$and $\mathcal{R}^{u p}{ }_{+}$, introduced in [4]. Recall that $S_{X}=\{x \in X:\|x\|=1\}$.

An operator $T \in \mathcal{B}(X, Y)$ belongs to $\mathcal{W}^{\text {up }}+$ if for every $0<r<1$ there exists $n \in \mathbb{N}$ for which there are no finite sets $\left\{x_{1}, \ldots, x_{n}\right\} \subset S_{X}$ and $\left\{f_{1}, \ldots, f_{n}\right\} \subset S_{X^{*}}$ for which $f_{i}\left(x_{j}\right)>r$ for $i \leq j, f_{i}\left(x_{j}\right)=0$ for $j<i$ and $\left\|T x_{k}\right\|<1 / k$, for $k=1, \ldots, n$. 
An operator $T \in \mathcal{B}(X, Y)$ belongs to $\mathcal{U}^{\text {up }}+$ if for every $C \geq 1$ there are $\delta>0$ and $n \in \mathbb{N}$ for which there is no finite set $\left\{x_{1}, \ldots, x_{n}\right\} \subset S_{X}$ which is $C$-equivalent to the unit basis of $\ell_{\infty}^{n}$ and satisfies $\left\|T x_{k}\right\|<\delta$ for $k=1, \ldots, n$.

An operator $T \in \mathcal{B}(X, Y)$ belongs to $\mathcal{R}^{u p}+$ if for every $C \geq 1$ there are $\delta>0$ and $n \in \mathbb{N}$ for which there is no finite set $\left\{x_{1}, \ldots, x_{n}\right\} \subset S_{X}$ which is $C$-equivalent to the unit basis of $\ell_{1}^{n}$ and satisfies $\left\|T x_{k}\right\|<\delta$ for $k=1, \ldots, n$.

Note that $T \in \mathcal{W}^{u p}+$ if and only if all the ultrapowers $T_{\mathfrak{U}}$ are tauberian operators [2]. Tacon [13] proved that $T \in \mathcal{W}^{u p}{ }_{+}$implies $T^{* *} \in \mathcal{W}^{u p}{ }_{+}$. Nevertheless, his proof does not seem to be applicable to the semigroups $\mathcal{U}^{u p}+$ and $\mathcal{R}^{u p}{ }_{+}$. Next we show that Theorem 10 allows us to give a unified proof of the result for the three semigroups $\mathcal{W}^{u p}{ }_{+}, \mathcal{R}^{u p}{ }_{+}$and $\mathcal{U}^{u p}{ }_{+}$.

Proposition 13. Let $\mathcal{A}_{+}$be any of the semigroups $\mathcal{W}^{u p}{ }_{+}, \mathcal{R}^{u p}{ }_{+}$or $\mathcal{U}^{u p}+$ and let $T \in \mathcal{B}(X, Y)$. Then $T \in \mathcal{A}_{+}$if and only if $T^{* *} \in \mathcal{A}_{+}$.

Proof. Let $\mathfrak{U}$ be an ultrafilter. It was proved in [2] (for $\mathcal{W}^{\text {up }}+$ ) and [4] (for $\mathcal{R}^{u p}+$ and $\left.\mathcal{U}^{u p}{ }_{+}\right)$that $T$ belongs to $\mathcal{A}_{+}$if and only if the zero operator $0_{\operatorname{ker}\left(T_{\mathfrak{U}}\right)}$ on $\operatorname{ker}\left(T_{\mathfrak{U}}\right)$ belongs to $\mathcal{A}_{+}$. In any of the three cases, the condition $0_{X} \in \mathcal{A}_{+}$defines a superproperty; i.e., $Y$ f.r. in $X$ and $0_{X} \in \mathcal{A}_{+}$implies $0_{Y} \in \mathcal{A}_{+}$.

By Theorem 10, $\operatorname{ker}\left(T^{* *} \mathfrak{U}\right)$ is f.r. in $\operatorname{ker}\left(T_{\mathfrak{U}}\right)$. Since $\operatorname{ker}\left(T^{* *} \mathfrak{U}\right)$ contains $\operatorname{ker}\left(T_{\mathfrak{U}}\right)$, we conclude that $T \in \mathcal{A}_{+}$if and only if $T^{* *} \in \mathcal{A}_{+}$.

Proposition 14. Let $\mathcal{A}_{+}$be any of the semigroups $\mathcal{W}^{u p}, \mathcal{R}^{u p}{ }_{+}$or $\mathcal{U}^{u p}{ }_{+}$ and let $T \in \mathcal{B}(X, Y)$. Then $T_{\mathfrak{U}}{ }^{*} \in \mathcal{A}_{+}$if and only if $T^{*} \mathfrak{U} \in \mathcal{A}_{+}$.

Proof. The direct implication is clear since $T_{\mathfrak{U}}{ }^{*}$ is an extension of $T^{*} \mathfrak{U}$. For the converse implication, observe that Theorem 11 says that $\operatorname{ker}\left(T_{\mathfrak{U}}{ }^{*}\right)$ is finitely representable in $\operatorname{ker}\left(T^{*} \mathfrak{U}\right)$. Now, it is enough to observe that $T^{*} \in$ $\mathcal{W}^{u p}+$ if and only if $\operatorname{ker}\left(T^{*} \mathfrak{U}\right)$ is superreflexive [2], and $T^{*}$ belongs to $\mathcal{R}^{u p}+$ (resp. $\mathcal{U}^{u p}{ }_{+}$) if and only if $\ell_{1}$ (resp. $c_{0}$ ) is not finitely representable in $\operatorname{ker}\left(T^{*} \mathfrak{U}\right)[\mathbf{4}]$.

The following result was proved by Tacon using nonstandard analysis. Here we give a more transparent proof.

Proposition 15 ([13, Theorem 3]). Given an operator $T \in \mathcal{B}(X, Y)$, we have $T^{*} \in \mathcal{W}^{\text {up }}+$ if and only if $\operatorname{ker}\left(T^{*} \mathfrak{U}\right)=\operatorname{ker}\left(T_{\mathfrak{U}}{ }^{*}\right)$.

Proof. By Corollary 12 we have that $\operatorname{ker}\left(T^{*} \mathfrak{U}\right)$ is $w^{*}$-dense in $\operatorname{ker}\left(T_{\mathfrak{U}}{ }^{*}\right)$. Moreover, it is shown in [2, Theorem 9] that $T^{*}$ belongs to $\mathcal{W}^{u p}+$ if and only if $\operatorname{ker}\left(T^{*} \mathfrak{U}\right)$ is reflexive. Hence the direct implication is clear.

Conversely, assume that $T^{*} \notin \mathcal{W}^{u p}{ }_{+}$; equivalently, $\operatorname{ker}\left(T^{*} \mathfrak{U}\right)$ is not reflexive. If $\operatorname{ker}\left(T_{\mathfrak{U}}{ }^{*}\right)=\operatorname{ker}\left(T^{*} \mathfrak{U}\right)$, since $\operatorname{ker}\left(T_{\mathfrak{U}}{ }^{*}\right)=\operatorname{coker}\left(T_{\mathfrak{U}}\right)^{*}$, the triangular 
arrays in non-reflexive spaces, discovered in [6] and [11], give normalized sequences $\left(\mathbf{y}_{n}+\overline{R\left(T_{\mathfrak{U}}\right)}\right)_{n}$ in $\operatorname{coker}\left(T_{\mathfrak{U}}\right)$ and $\left(\mathbf{f}_{n}\right)$ in $\operatorname{ker}\left(T^{*} \mathfrak{U}\right)$, and $0<\varepsilon<1$ so that

$$
\mathbf{f}_{k}\left(\mathbf{y}_{l}\right)= \begin{cases}>\varepsilon, & \text { if } 1 \leq k \leq l<\infty \\ =0, & \text { if } 1 \leq l<k<\infty .\end{cases}
$$

Write $\mathbf{y}_{n}=\left[y_{n}^{i}\right]_{i}$ and $\mathbf{f}_{n}=\left[f_{n}^{i}\right]_{i}$. Let $\mathbf{g}=\left[g_{i}\right]$ be a $w^{*}$-cluster point of $\left\{\mathbf{f}_{n}: n \in \mathbb{N}\right\}$ in $\operatorname{ker}\left(T^{*} \mathfrak{U}\right)$. Formula 4 yields $\mathbf{g}\left(\mathbf{y}_{n}\right)=0$ for all $n \in \mathbb{N}$, and allows us to build the sequence $\left(A_{n}\right)_{n}$ of elements of $\mathfrak{U}$ given inductively by

$$
\begin{aligned}
& A_{1}:=\left\{i \in I: g_{i}\left(y_{1}^{i}\right)<\varepsilon / 2, f_{1}^{i}\left(y_{1}^{i}\right)>\varepsilon\right\} \in \mathfrak{U} \\
& A_{n}:=A_{n-1} \cap\left\{i \in I: g_{i}\left(y_{n}^{i}\right)<\varepsilon / 2, f_{k}^{i}\left(y_{n}^{i}\right)>\varepsilon, 1 \leq k \leq n\right\} \in \mathfrak{U} .
\end{aligned}
$$

Since $\left(A_{n}\right)_{n}$ is decreasing, by Lemma 9 we find another decreasing sequence $\left(C_{n}\right)_{n} \subset \mathfrak{U}$ such that $C_{n} \subset A_{n}$ and $C_{n} \backslash C_{n+1} \neq \emptyset$ for all $n \in \mathbb{N}$, and $\bigcap_{n=1}^{\infty} C_{n}=\emptyset$. Thus, for every $k \in \mathbb{N}$, we take $s_{i}:=y_{k}^{i}$ for every $i \in C_{k} \backslash C_{k+1}$, and define $\mathbf{s}:=\left[s_{i}\right]$.

On the one hand, we have that $\mathbf{g}(\mathbf{s}) \geq \varepsilon$. Indeed, let $k$ be a positive integer. For every $l>k$ and every $i \in C_{l} \backslash C_{l+1}$ we have $f_{k}^{i}\left(s_{i}\right)=f_{k}^{i}\left(y_{l}^{i}\right) \geq \varepsilon$. Since $\bigcup_{l=k}^{\infty}\left(C_{l} \backslash C_{l+1}\right) \in \mathfrak{U}$, we have $\left[f_{k}^{i}\right]\left(\left[s_{i}\right]\right) \geq \varepsilon$; therefore, $\mathbf{f}_{k}(\mathbf{s}) \geq \varepsilon$ for each $k \in \mathbb{N}$. Besides that, as $\mathbf{g}$ is a $w^{*}$-cluster point of $\left\{\mathbf{f}_{n}: n \in \mathbb{N}\right\}$, we have $\mathbf{g}(\mathbf{s}) \geq \varepsilon$.

On the other hand, for every $n \in \mathbb{N}$ and $i \in C_{n} \backslash C_{n+1} \subset A_{n}$ we have that $g_{i}\left(s_{i}\right)=g_{i}\left(y_{n}^{i}\right)<\varepsilon / 2$. Thus $\bigcup_{n=1}^{\infty}\left(C_{n} \backslash C_{n+1}\right) \in \mathfrak{U}$ leads to $\mathbf{g}(\mathbf{s})=\left[g_{i}\right]\left(\left[s_{i}\right]\right) \leq$ $\varepsilon / 2$, and we get a contradiction.

Acknowledgments. Work on this paper was completed while the second author was visiting the Department of Mathematics of the University of Texas at Austin during the academic year 1996-97. He would like to express his gratitude for the hospitality offered to him by its Banach space Theory team. In particular, he would like to thank G. Androulakis, E. Odell and H. Rosenthal for helpful discussions.

Both authors thank N. Kalton for the example included in the Remark after Theorem 4.

\section{References}

[1] G. Godefroy, Existence and uniqueness of isometric preduals: A survey, Contemporary Math., 85 (1989), 131-190.

[2] M. González and A. Martínez-Abejón, Supertauberian operators and perturbations, Arch. Math., 64 (1995), 423-433.

[3] _ Ultrapowers and semi-Fredholm operators, Bollettino U.M.I., 11-B (1997), 415-433.

$[4]$ Ultrapowers and semigroups of operators, Preprint. 
[5] S. Heinrich, Ultraproducts in Banach space theory, J. Reine Angew. Math., 313 (1980), 72-104.

[6] R.C. James, Characterizations of reflexivity, Studia Math., 23 (1964), 205-216.

[7] N. Kalton and A. Wilansky, Tauberian operators on Banach spaces, Proc. Amer. Soc., 57 (1976), 251-255.

[8] J. Lindenstrauss and H. Rosenthal, The $\mathcal{L}_{p}$ spaces, Israel J. Math., 7 (1969), 325-349.

[9] A. Martínez-Abejón, Semigrupos de operadores y ultrapotencias, Doctoral Thesis, Universidad de Cantabria, 1994.

[10] _ An elementary proof of the principle of local reflexivity, Proc. Amer. Math. Soc., to appear.

[11] D.P. Milman and V.D. Milman, The geometry of nested families with empty intersection. The structure of the unit sphere of a non-reflexive space, Matem. Sbornik, 66 (1965), 109-118.

[12] D.G. Tacon, Generalized semi-Fredholm transformations, J. Austral Math. Soc., 34 (1983), 60-70.

[13] _ Generalized Fredholm transformations, J. Austral Math. Soc., 37 (1984), 89-97.

Received October 1, 1997 and revised February 24, 1998. This paper was supported in part by DGICYT Grant PB 94-1052 (Spain). The second author was supported by a postdoctoral Grant of the Ministry of Spain for Education and Science.

Universidad de Cantabria

39071 SANTANDER

SPAIN

E-mail address: gonzalem@ccaix3.unican.es

UNIVERSIDAD DE OVIEDO

33007 OvIEDO

SPAIN

E-mail address: ama@pinon.ccu.uniovi.es 